\title{
Institutional Review Board Independent Ethics Committee Human Subject in Research Approval Documentation
}

National Cancer Institute

\section{Source}

National Cancer Institute. Institutional Review Board Independent Ethics Committee

Human Subject in Research Approval Documentation. NCI Thesaurus. Code C115696.

Records pertaining to site specific approvals from an Institutional Review Board

(IRB)/Independent Ethics Committee (IEC) or other governing body regarding human

subjects in a biomedical or behavioral research. 